\title{
Numerical predictions of U-notched sample failure based on a discrete energy argument
}

Zahedi, S. A.; Kodsi, Costy; Berto, F.

Published in:

Theoretical and Applied Fracture Mechanics

Link to article, DOI:

10.1016/j.tafmec.2018.12.014

Publication date:

2019

Document Version

Peer reviewed version

Link back to DTU Orbit

Citation (APA):

Zahedi, S. A., Kodsi, C., \& Berto, F. (2019). Numerical predictions of U-notched sample failure based on a discrete energy argument. Theoretical and Applied Fracture Mechanics, 100, 298-306.

https://doi.org/10.1016/j.tafmec.2018.12.014

\section{General rights}

Copyright and moral rights for the publications made accessible in the public portal are retained by the authors and/or other copyright owners and it is a condition of accessing publications that users recognise and abide by the legal requirements associated with these rights.

- Users may download and print one copy of any publication from the public portal for the purpose of private study or research.

- You may not further distribute the material or use it for any profit-making activity or commercial gain

- You may freely distribute the URL identifying the publication in the public portal

If you believe that this document breaches copyright please contact us providing details, and we will remove access to the work immediately and investigate your claim. 


\title{
Numerical predictions of U-notched sample failure based on a discrete energy argument
}

\author{
S. A. Zahedi ${ }^{\mathrm{a}, *}$, C. Kodsi ${ }^{\mathrm{b}}$, F. Berto ${ }^{\mathrm{c}}$ \\ ${ }^{a}$ School of Engineering and Sustainable Development, De Montfort University, Gateway House, Leicester LE1 9BH, U.K. \\ ${ }^{b}$ Department of Applied Mathematics and Computer Science, Technical University of Denmark, 2800 Kgs. Lyngby, Denmark \\ ${ }^{c}$ Department of Engineering Design and Materials, NTNU, Richard Birkelands vei 2b, 7491 Trondheim, Norway
}

\begin{abstract}
Kodsi (2016) proposed a criterion for isotropic, linear elastic media in a brittle state applicable to both sharp and blunt notches. Fracture toughness and material strength are the only parameters required to operate the criterion. This paper provides a formulation of the criterion specific to blunt notches and further examines the capability of the criterion for samples weakened by U-notches. Predictions are compared to significant experimental results from U-notched samples that were subject to mixed-mode fracture. Good agreement is demonstrated, justifying and substantiating the theory.
\end{abstract}

Keywords: Brittle fracture, Crack onset, Fracture criterion, Failure predictions, U-notched samples

\section{Introduction}

Extensive experimental data related to the static failure of vertical and tilted U-notched polymethylmetacrylate (PMMA) samples was supplied by Gómez et al. in [1] and [2]. The tests were designed to induce mixed-mode (I-II) fracture in the samples with cracks emanating from the U-notches. In [1], the average strain energy density of a small crescent-shaped control area local to the notch was computed and used to provide critical (fracture) load predictions. But first, however, the control area had to be centred at the point of maximum principal stress present on the notch edge. This proved to be a decent indicator of crack onset location. Predictions made by the average strain energy density criterion compared well with experimental results. Still, there were two steps involved in forming a prediction for the critical load. Furthermore, the criterion itself is not part of a physical theory or mathematical description justifying the computation of the strain energy density mean value over a given area. Application of the established cohesive process zone concept $[3,4]$ to predict the critical load for crack propagation from the U-notches yielded excellent results $[2,5]$. Again, the maximum principal stress was taken as the location of crack onset. Implementation of such models is not so straightforward. Firstly, the form of the softening function must be inferred. Secondly, and probably the most difficult to contend with, is the numerical realisation. Propagation direction of a crack must be known a priori and the geometrical discretisation, for example in the finite element method, has to be carefully designed so that cohesive elements lie along the path.

Novozhilov made an astute observation in [6] that fracture in solids is a discrete process, that is, considering the atomic make-up of matter. A non-local stress criterion was put forward by Novozhilov, in which fracture occurs when the mean value of de-cohesive stress taken over an elementary increment reaches a critical value. This elementary increment or fracture quantum [7] represented the diameter of the atom and the stress was acting in the normal direction to an atomic layer. Seweryn and Mróz [8] generalised the criterion to predict crack propagation from notches subject to mixed-mode deformation. The increment was still treated as a material characteristic but now based on linear elastic fracture mechanics (LEFM). The

\footnotetext{
* Corresponding author

Email address: abolfazl.zahedi@dmu.ac.uk (S. A. Zahedi)
} 


\section{Nomenclature}

$\begin{array}{ll}a_{c} & \text { increment length } \\ b & \text { thickness of U-notched samples } \\ E & \text { Young's modulus } \\ g & \text { distance of applied load from centre } \\ G_{c} & \text { critical energy release rate } \\ h & \text { U-notch height } \\ K_{I c} & \text { (mode-I) fracture toughness } \\ l & \text { length of U-notched samples } \\ m & \text { distance of support from centre } \\ P & \text { applied load } \\ Q & \text { change in energy } \\ w & \text { width of U-notched samples } \\ Y & \text { non-dimensional constant } \\ \nu & \text { Poisson's ratio } \\ \rho & \text { U-notch root radius } \\ \sigma & \text { Cauchy stress } \\ \sigma_{c} & \text { (material) tensile strength } \\ \varphi & \text { crack onset angle } \\ \xi_{c} & \text { virtual (small) circle domain radius }\end{array}$

Acronyms

LEFM linear elastic fracture mechanics

PMMA polymethyl-metacrylate

capability of the formulation was demonstrated on V-notched samples that were subjected to multi-axial loading $[9,10]$. An energy-based equivalent to the non-local stress criterion was developed by Pugno and Ruoff [7] as part of their quantised fracture mechanics.

Based on Novozhilov's and successive theories, Kodsi [11, 12] took the viewpoint that crack nucleation at the macroscopic scale in isotropic, linear elastic and brittle media is a sudden and discrete phenomenon. Accordingly, he cast Griffith's criterion [13] into a finite difference form, as in [7]. Crack nucleation, thus, only results if the change in total potential energy is equal to the energy spent on creating the finite-length crack, which is given by the critical energy release rate together with the increment length. The increment length was obtained from Pugno and Ruoff's energy-based equivalent to the non-local stress criterion by bounding the stress for a vanishing (edge) crack in an infinite plate subject to tensile loading. An asymptotic analysis was employed to derive a closed-form expression for the change in total potential energy due to the introduction of a small circular hole at a point (being the hole centre) in the unperturbed, i.e., virgin domain. To determine the size of the hole, a calibration scenario of mode-I fracture was studied. A distinguishing feature of this approach is generality of application; crack propagation can be predicted from both sharp and blunt notches. Moreover, the criterion is readily automated and can be easily integrated into the postprocessing framework of a finite element analysis software for example. Only the fracture toughness and material strength are required for criterion operation.

Validation of the criterion involved the prediction of pure and mixed-mode fracture in notched PMMA samples; numerous circular [14] notched, U-notched [1] and V-notched [9] samples were scrutinised. The results were very supportive of the theory. This work (i) provides a version of the criterion specific to blunt notches, (ii) presents the theory behind the criterion in a manner befitting an engineering audience, and (iii) rigorously examines the predictive capability of the criterion focusing on samples weakened by U-notches. 
To that end, experimental tests in [1] and [2] are modelled numerically and predictions by the criterion are solicited. This is an expanded set of tests compared to [11, 12] and serves the purpose of not only corroborating Kodsi's findings but also for furthering confidence in the criterion.

The paper is organised as follows. In Section 2, the criterion proposed by Kodsi is concisely reviewed. Then the experimental tests in [1] and [2] are summarised in Section 3. Crack onset location and critical load predictions are compared with experimental results in Section 4. Section 5 concludes this work.

\section{Discrete energy approach}

Crack propagation according to the proposed criterion is assumed to occur when the energy, $Q$, associated with the creation of a small and finite-length crack satisfies a critical value:

$$
Q=a_{c} G_{c}
$$

in which $a_{c}$ is a (material) incremental length and $G_{c}$ is the critical energy release rate. While the latter is experimentally derived, the former is defined (refer to $[11,12]$ for the derivation) as

$$
a_{c}=\frac{2}{\pi}\left(\frac{K_{I c}}{Y \sigma_{c}}\right)^{2},
$$

where $K_{I c}$ is the (mode-I) fracture toughness, $Y=1.1215$ and $\sigma_{c}$ is the tensile strength.

The change in energy due to finite-length crack propagation is assessed at points that are a distance $\xi_{c}$ in the inward normal direction to the body domain boundary, see Figure 1a, by

$$
Q=\frac{\pi \xi_{c}^{2}}{2 \bar{E}}\left(3 \sigma_{x x}^{2}+3 \sigma_{y y}^{2}-2 \sigma_{x x} \sigma_{y y}+8 \sigma_{x y}^{2}\right)
$$

where $\bar{E}=E$ for plane stress and $\bar{E}=E /\left(1-\nu^{2}\right)$ for plane strain. Here, $\sigma_{i j}$ represents components of the Cauchy stress tensor in the standard Cartesian coordinate system. This expression, in fact, provides the approximate energy change associated with the perforation of a domain, shown in Figure 1b, by a small circular hole. Equivalence of the energy change to a discrete crack extension event is achieved through the hole radius $\xi_{c}$.

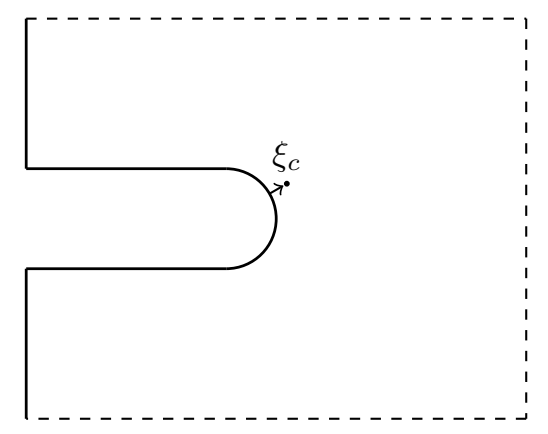

(a)

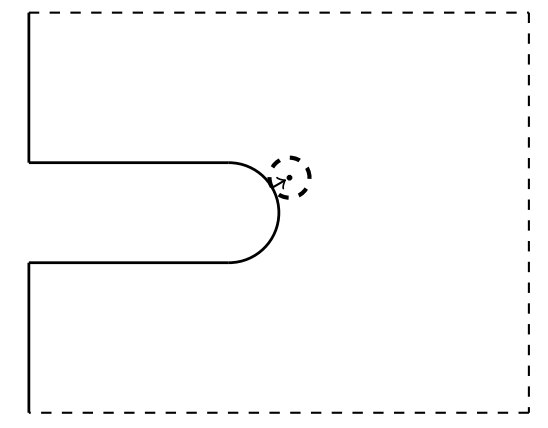

(b)

Figure 1. Energy change, (a) evaluated at points a distance $\xi_{c}$ in the inward normal direction to the boundary, associated with a (b) small circular domain perforation.

In order to surmise the form of $\xi_{c}$, mode-I fracture of a double-edge semi-circular notched sample is considered. Crack onset location is known, so is the propagation direction, and an assumption of (relatively) uniform stress ahead of the notch root bounded by the tensile strength can be made. Subsequently, $\sigma_{y y}=\sigma_{c}$ and $\sigma_{x x}=\sigma_{x y}=0$, leading to

$$
\xi_{c}=\sqrt{\frac{2 a_{c} G_{c} \bar{E}}{3 \pi \sigma_{c}^{2}}} .
$$


If $\sigma_{x x}=\sigma_{c}$ and $\sigma_{y y}=\sigma_{x y}=0$, the result is consistent. For plane strain, this simplifies to

$$
\xi_{c}=\frac{2}{\sqrt{3} \pi Y}\left(\frac{K_{I c}}{\sigma_{c}}\right)^{2} .
$$

It is worth emphasising that the radius is taken as constant for any mode mixity prediction, i.e., the size of the small circular hole is fixed. There is no reason why $\xi_{c}$ could not be based on an alternate mixed-mode fracture scenario. However, calibration would be a more involved process and certainly not as simple as the employed strategy. To date, there has been no research conducted in this area. Even though the criterion itself is just as valid for sharp notches, the parameter $\xi_{c}$ must be set accordingly. The form of $\xi_{c}$ given here is only appropriate for blunt notches.

Once a linear elasticity boundary value problem solution is available, e.g. from a finite element analysis, the change in energy can be interrogated at points a distance $\xi_{c}$ from the boundary. Then the location of crack onset and critical load can be determined if the criterion is satisfied at one of the queried points.

\section{Experiments}

\subsection{Sample material properties}

PMMA, an amorphous glassy polymer, exhibits brittle characteristics and behaves in a near-linear elastic manner at $-60^{\circ} \mathrm{C}[4]$. Therefore, it is a popular choice in experiments for validating criteria based on the principles of linear elastic fracture mechanics. Mechanical properties of the PMMA samples tested in [1] and [2] are:

- Young's modulus, $E=5.05 \pm 0.04 \mathrm{GPa}$

- Poisson's ratio, $\nu=0.40 \pm 0.01$

- Tensile strength, $\sigma_{c}=128.4 \pm 0.1 \mathrm{MPa}$

- Fracture toughness, $K_{I c}=1.7 \pm 0.1 \mathrm{MPa} \sqrt{\mathrm{m}}$

\subsection{Sample geometry and loading}

Experimental data in [1] and [2] was reported for rectangular parallelepiped samples with vertical and $\left(45^{\circ}\right)$ tilted U-notches subjected to three point bend tests. All samples share the same length $l=126 \mathrm{~mm}$, width $w=28 \mathrm{~mm}$, thickness $b=14 \mathrm{~mm}$ and notch height $h=14 \mathrm{~mm}$. The support as well as loading positions for the vertical and tilted U-notched PMMA samples are depicted in Figure 2.

Eccentric loading of the vertical U-notched samples with fixed supports was used to induce mixed-mode fracture. Six loading positions were studied at distances $g=-3,3,9,18,27$ and $36 \mathrm{~mm}$ from the centre. The influence of the U-notch root radius at every loading position was also investigated: $\rho=0,0.2,0.3,0.5,1.0,2.0$ and $4.0 \mathrm{~mm}$. For $g=-3$ and $3 \mathrm{~mm}$, the radius corresponding to $0.2 \mathrm{~mm}$ was not considered.

Mixed mode fracture in the tilted samples was facilitated by varying the distance, $m$, of a support from the centre. Three values were analysed: $m=3,9$ and $15 \mathrm{~mm}$. As in the vertical U-notched case, the influence of the following U-notch root radii $\rho=0.3,0.5,1.0,2.0$ and $4.0 \mathrm{~mm}$ was explored for every value of $m$. Every test, whether vertical or tilted, was repeated three times.

\subsection{Testing procedure}

Testing took place on a servo-controlled INSTRON machine. The experimental set-up was located inside an INSTRON environmental chamber. First, the sample was cooled to $-60^{\circ} \mathrm{C}$ through the use of liquid nitrogen over a thirty minute period and a small pre-load was applied (under load control). Once the sample temperature was stabilised (remaining under load control), displacement control testing was performed at constant temperature at a rate of $0.05 \mathrm{~mm} / \mathrm{min}$ for the vertical U-notched samples with loading positions $g=9,18,27$ and $36 \mathrm{~mm}$ and $0.03 \mathrm{~mm} / \mathrm{min}$ for the others. Results of the vertical $(\rho=0$ and $0.2 \mathrm{~mm}$ omitted $)$ and tilted U-notched PMMA experiments are included in Appendix A for completeness. Location of crack onset on the notch is given by the angle $\varphi$ measured from the vertical axis, see Figure 3 . 


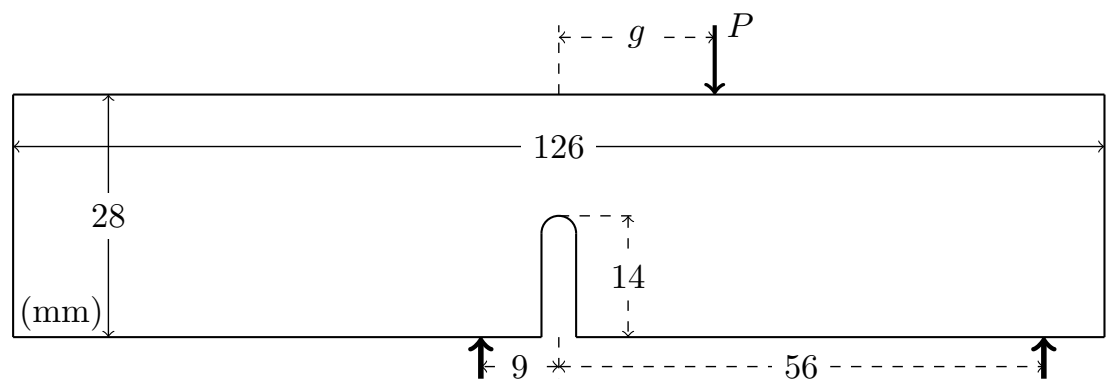

(a)

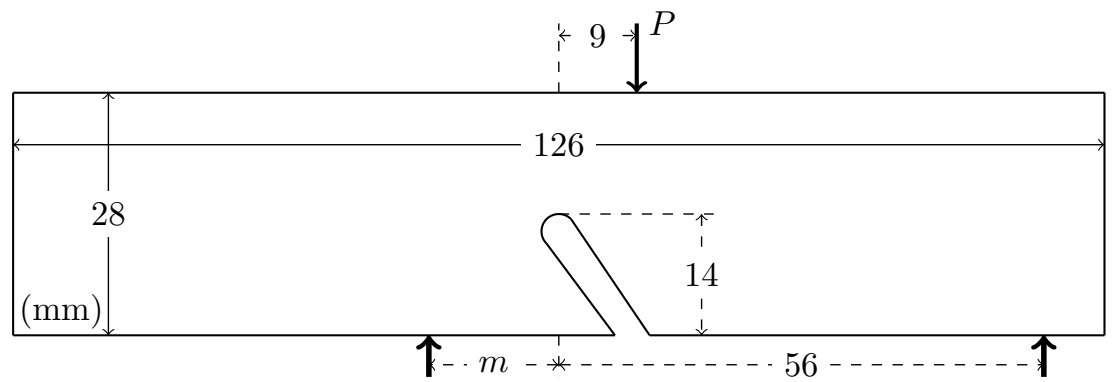

(b)

Figure 2. (a) Vertical and (b) tilted U-notched sample test configurations; Thickness $=14 \mathrm{~mm}$.

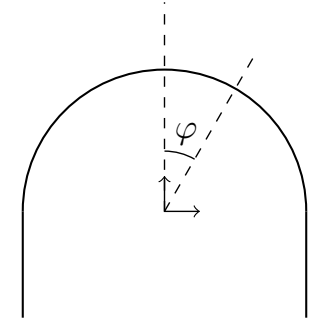

Figure 3. Crack onset location, angle $\varphi$.

\section{Numerical predictions}

\subsection{Model}

Stress in the tested samples was obtained numerically by means of the open source Code_Aster [15] finite element package. All the analyses were carried out under the hypothesis of linear elasticity and an assumption of plane strain. Code_Aster has the ability to handle point loads and supports. This is advantageous since the imposed boundary conditions are all of this variety. A highly refined mesh composed of quadratic quadrilateral finite elements was employed in the vicinity of the U-notch tip region. In order to gauge the level of refinement, a mesh-sensitivity study was undertaken. Over one hundred thousand elements was the standard. Unfortunately, the analysis of tilted U-notched samples corresponding to $\rho=0.3$ and $0.5 \mathrm{~mm}$ proved to be too computationally onerous and were abandoned. In order to evaluate the criterion in question, a subroutine was developed for interpolating the stress around the U-notch tip involving nodal value projections. Also, due to the computing power, the angle representing crack onset location was only examined at one degree intervals. For the assessment of fracture, $a_{c}=8.873 \times 10^{-5} \mathrm{~m}$ and $\xi_{c}=5.745 \times 10^{-5} \mathrm{~m}$. 


\subsection{Vertical U-notched samples}

Location of crack onset and critical load predictions are plotted along with experimental results against the notch root radii for $g=-3,3,9,18,27$ and $36 \mathrm{~mm}$ in Figures 4-9. Even though the predictive angle for crack onset location is impacted (most pronounced in Figures 8 and 9) by the aforementioned restriction, the trends are captured exceptionally well in all cases if $\rho=0.3 \mathrm{~mm}$ with respect to $g=3,18,27$ and $36 \mathrm{~mm}$ is discounted or ignored. This is wholly justified given the rather capricious nature of these results, as can be more clearly observed in $[11,12]$. It can be thus concluded that there is very good agreement between the predictions and experimental results. Predictions of the critical loads are sound and apart from $\rho=2 \mathrm{~mm}$ at $g=-3,3 \mathrm{~mm}$ as well as $\rho=4 \mathrm{~mm}$ at $g=3,18 \mathrm{~mm}$ are consistently a little conservative. Trends are, however, captured quite well. The predictions for $g=9,18,27$ and $36 \mathrm{~mm}$ are in line with those reported in $[11,12]$.
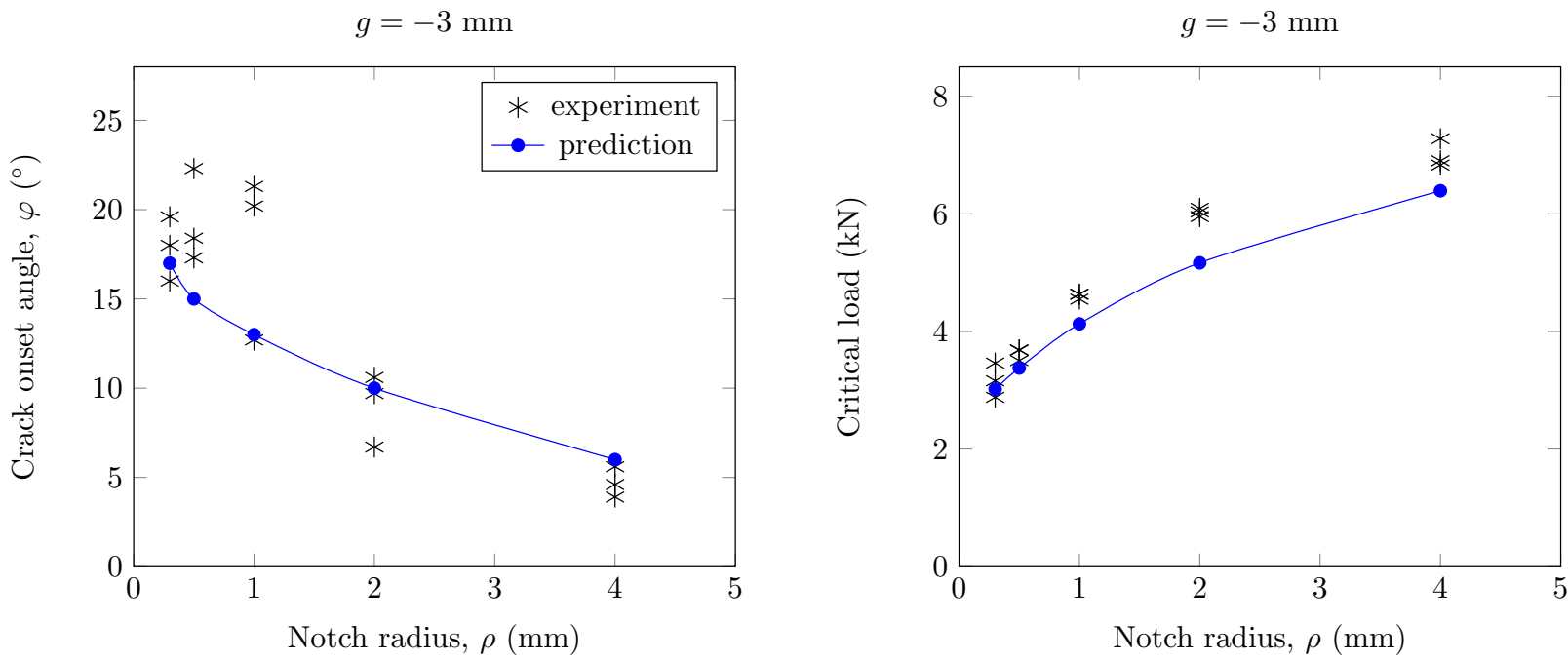

Figure 4. Experimental results and numerical predictions for $g=-3 \mathrm{~mm}$.
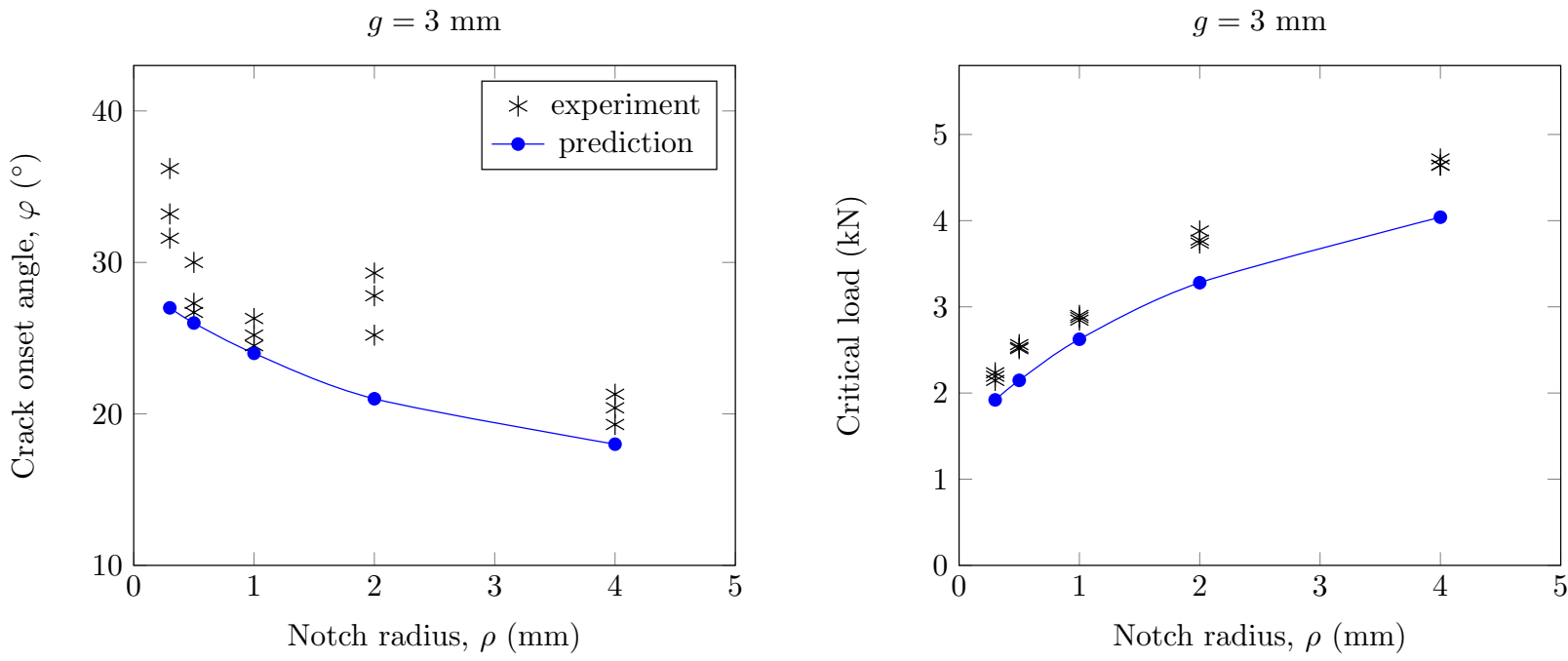

Figure 5. Experimental results and numerical predictions for $g=3 \mathrm{~mm}$. 

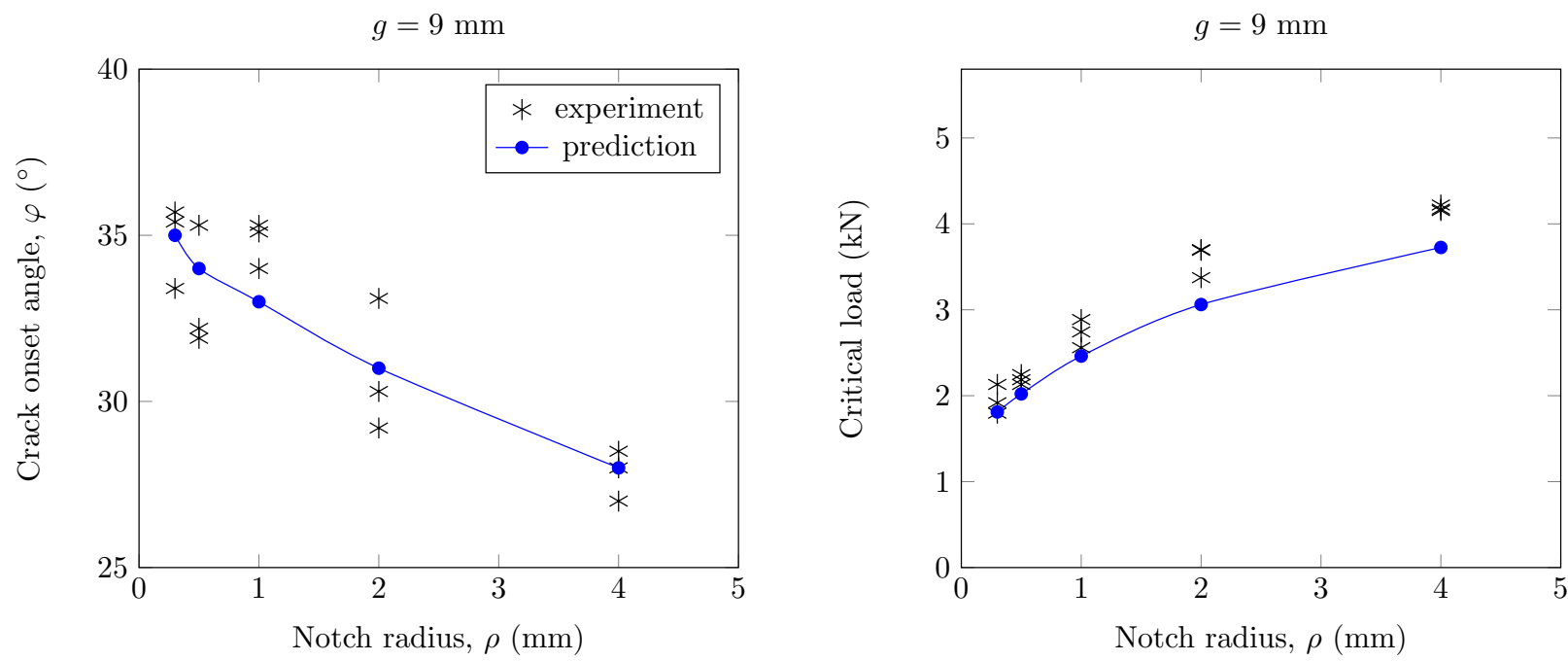

Figure 6. Experimental results and numerical predictions for $g=9 \mathrm{~mm}$.
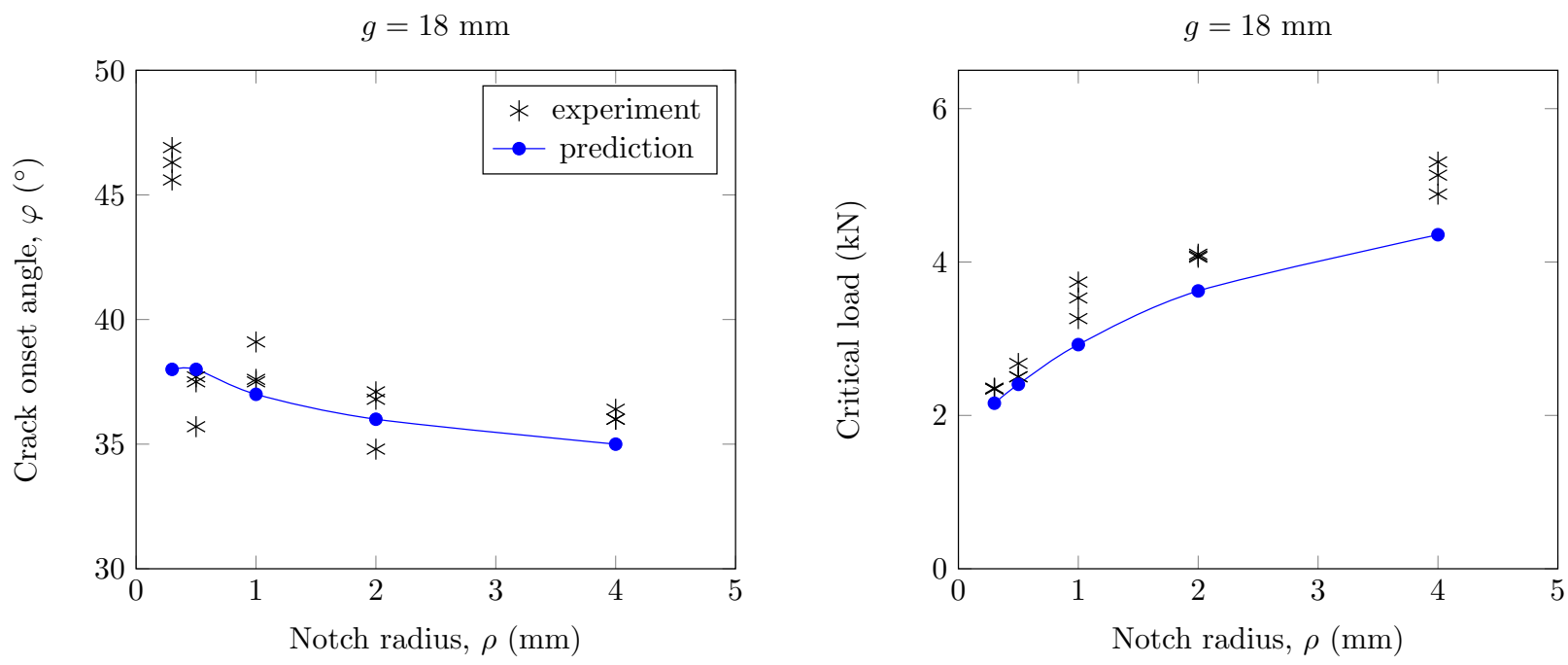

Figure 7. Experimental results and numerical predictions for $g=18 \mathrm{~mm}$.

\subsection{Tilted U-notched samples}

Once again, location of crack onset and critical load predictions are plotted along with experimental results against the notch root radii for $m=3,9$ and $15 \mathrm{~mm}$ in Figures 10-12. As in the vertical U-notch case, the criterion offers critical load predictions that capture the trends but are just shy of the experimental results (exceptions being $\rho=2 \mathrm{~mm}$ at $m=3 \mathrm{~mm}$ and $\rho=4 \mathrm{~mm}$ at $m=15 \mathrm{~mm}$ ). It is a bit more of a complicated matter with regards to the crack onset location. While the predicted angles are generally close to or within the scatter of experimental results, the trends (including results for $\rho=0.3$ and $0.5 \mathrm{~mm}$ ) are not so obvious or sufficiently clear to arrive at a firm conclusion one way or the other. A numerical investigation of the maximum principal stress on the notch edge that can be found in [16], however, supports the resolved angles. 

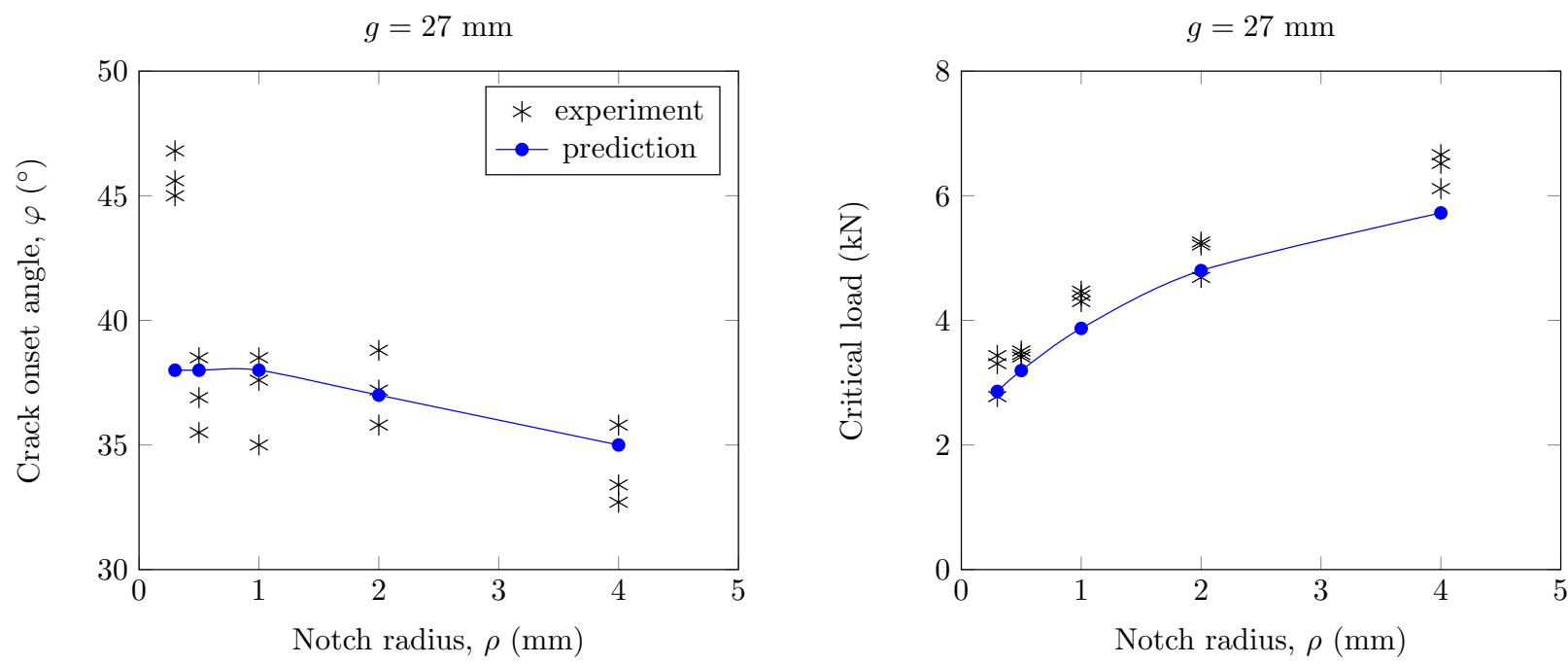

Figure 8. Experimental results and numerical predictions for $g=27 \mathrm{~mm}$.
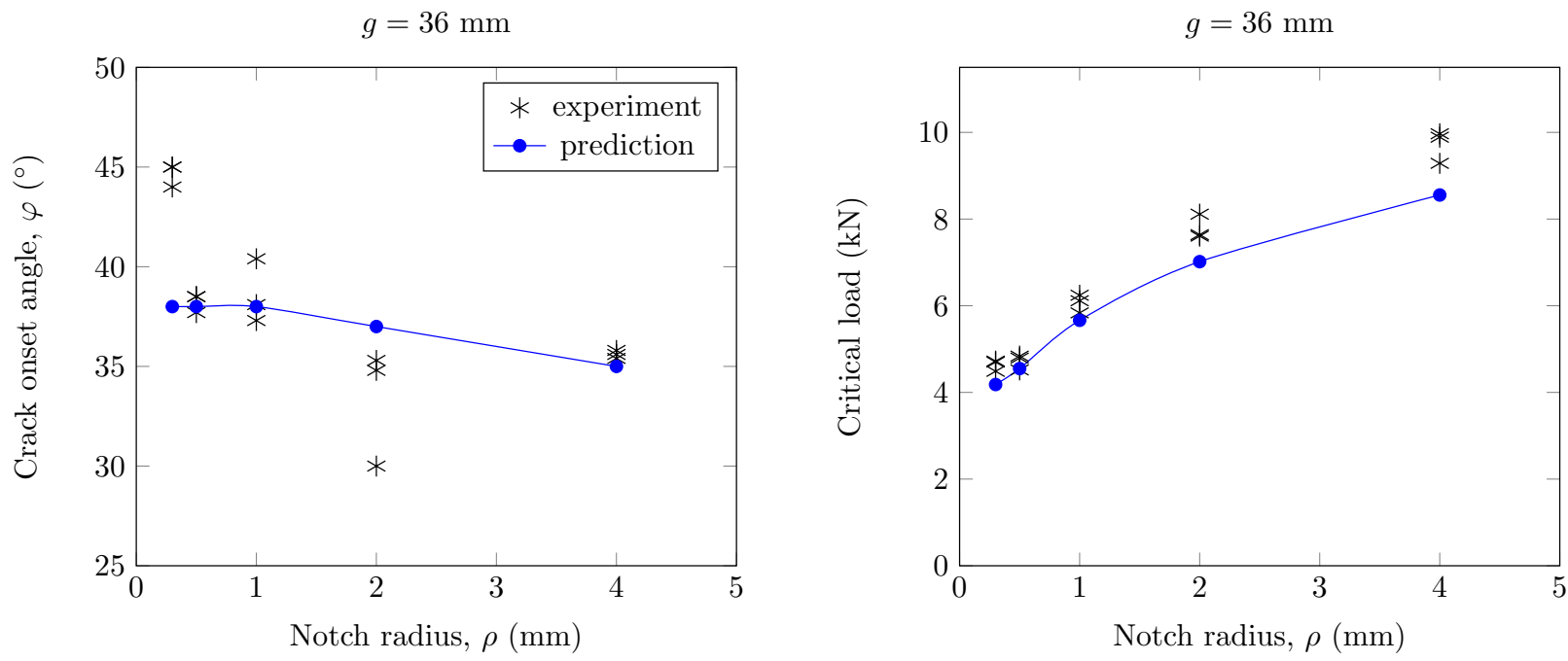

Figure 9. Experimental results and numerical predictions for $g=36 \mathrm{~mm}$.

\section{Conclusions}

Predictions made by the criterion proposed by Kodsi $[11,12]$ have been compared to extensive experimental results from U-notched PMMA samples that were subjected to three-point bend testing. Mixed-mode fracture, in the tests, was induced through a non-symmetric arrangement of supports and loading. It was comprehensively shown that the criterion provides very reliable predictions. There was very good agreement between the predicted and experimental results for crack onset location. Critical loads were very close to the experimental results but slightly conservative, though not by a sufficient amount to detract from the quality of the prediction. This work gives further credence to the criterion. Moreover, the presentation of the theory should, hopefully, be more amenable to the engineering community. Analysis of U-notched disc-type graphite samples [17] would be an interesting avenue of research to pursue next. 

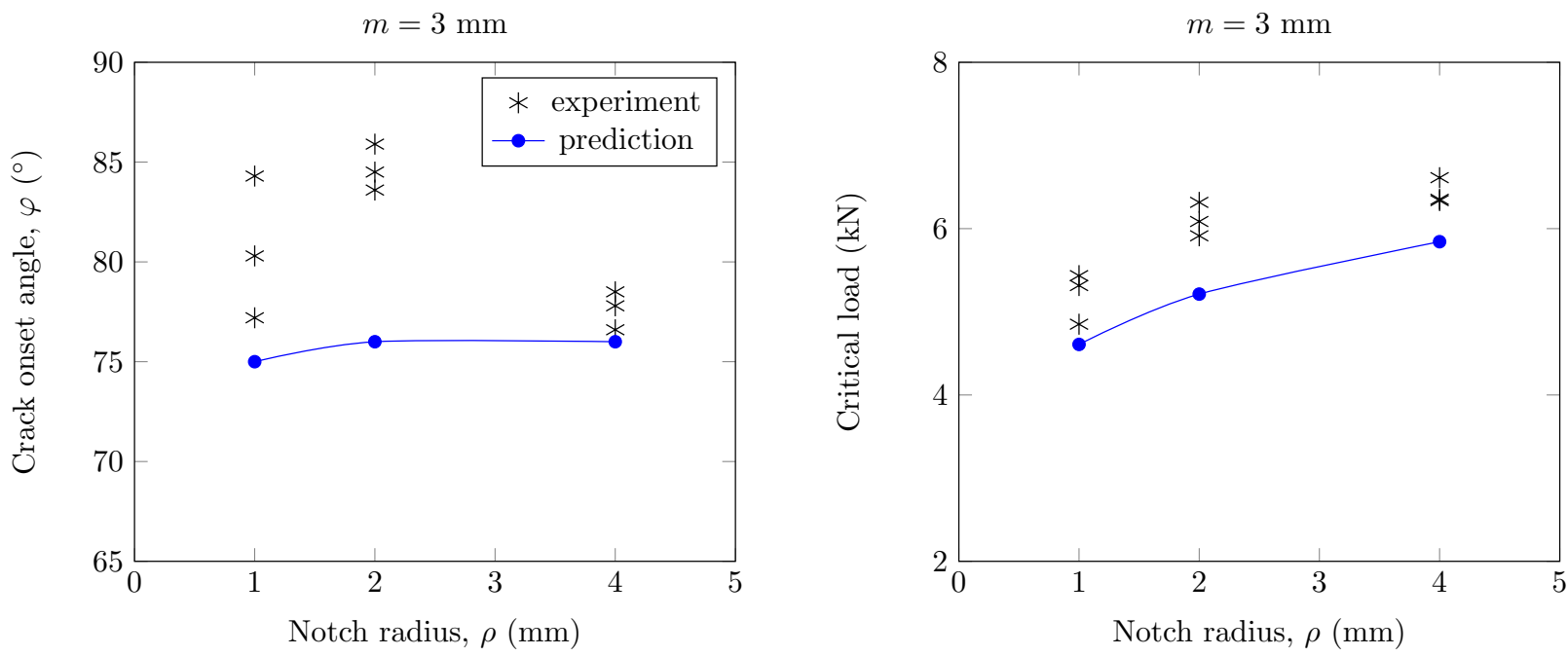

Figure 10. Experimental results and numerical predictions for $m=3 \mathrm{~mm}$.
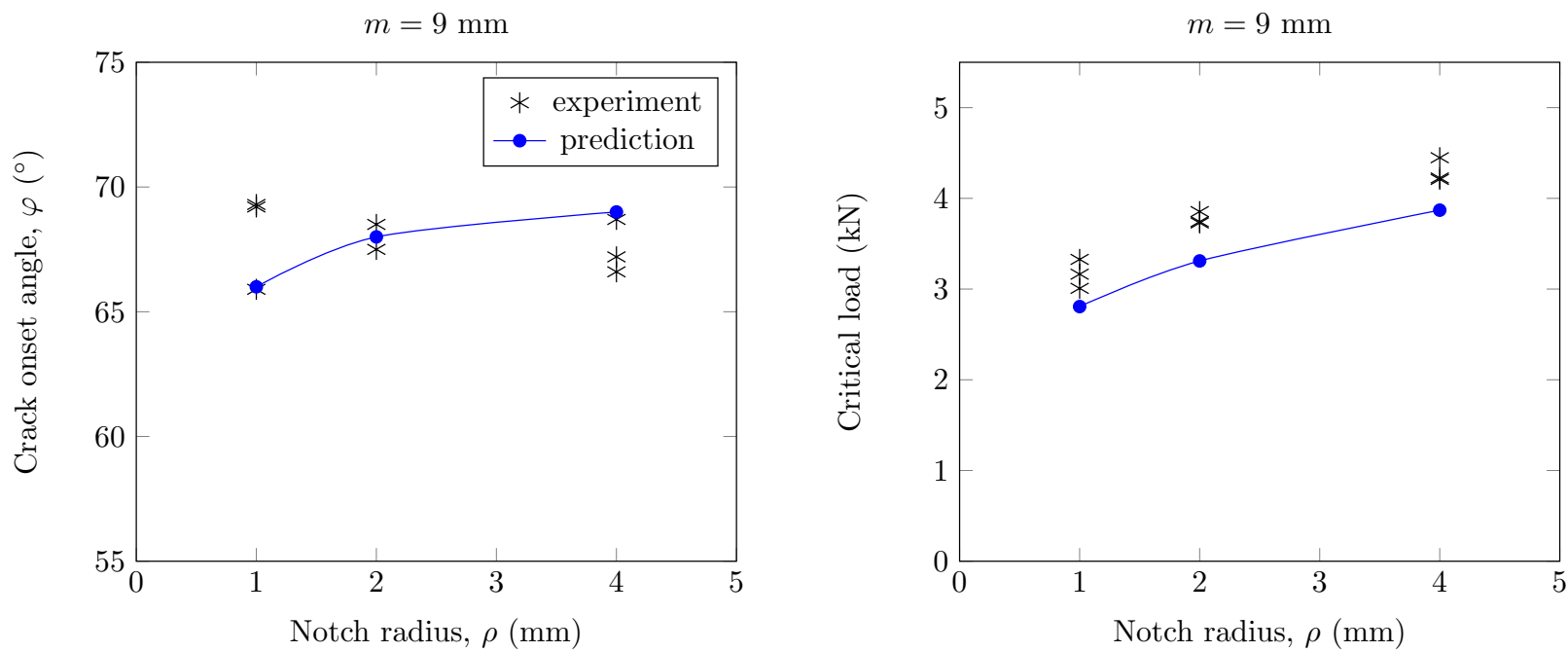

Figure 11. Experimental results and numerical predictions for $m=9 \mathrm{~mm}$.

\section{Acknowledgement}

The second author is grateful for the financial support received from the European Research Council under the European Union's Horizon 2020 research and innovation programme (grant agreement no. 757360).

\section{Appendix A. Experimental results}

Table A.1. Vertical U-notched samples (reproduced from [2]).

\begin{tabular}{cccccccccc}
\hline$\rho(\mathrm{mm})$ & $g(\mathrm{~mm})$ & $P_{1}(\mathrm{~N})$ & $P_{2}(\mathrm{~N})$ & $P_{3}(\mathrm{~N})$ & $\langle P\rangle(\mathrm{N})$ & $\varphi_{1}^{\circ}$ & $\varphi_{2}^{\circ}$ & $\varphi_{3}^{\circ}$ & $\langle\varphi\rangle^{\circ}$ \\
\hline 4 & -3 & 6903 & 6830 & 7277 & 7003 & 3.9 & 5.6 & 4.6 & 4.7 \\
& 3 & 4716 & 4643 & 4645 & 4668 & 19.3 & 21.3 & 20.4 & 20.3 \\
& 4 & 4219 & 4155 & 4171 & 4182 & 28.0 & 27.0 & 28.5 & 27.8 \\
\hline & & & & & Continued on next page
\end{tabular}


Table A.1 - Continued from previous page

\begin{tabular}{|c|c|c|c|c|c|c|c|c|c|}
\hline$\rho(\mathrm{mm})$ & $g(\mathrm{~mm})$ & $P_{1}(\mathrm{~N})$ & $P_{2}(\mathrm{~N})$ & $P_{3}(\mathrm{~N})$ & $\langle P\rangle(\mathrm{N})$ & $\varphi_{1}^{\circ}$ & $\varphi_{2}^{\circ}$ & $\varphi_{3}^{\circ}$ & $\langle\varphi\rangle^{\circ}$ \\
\hline & 18 & 4885 & 5304 & 5133 & 5107 & 36.0 & 36.4 & 36.0 & 36.1 \\
\hline & 27 & 6114 & 6518 & 6659 & 6430 & 35.8 & 32.7 & 33.4 & 34.0 \\
\hline & 36 & 9968 & 9889 & 9286 & 9714 & 35.4 & 35.8 & 35.6 & 35.6 \\
\hline \multirow[t]{6}{*}{2} & -3 & 5954 & 6093 & 6033 & 6027 & 6.7 & 10.6 & 9.7 & 9.0 \\
\hline & 3 & 3880 & 3740 & 3775 & 3798 & 25.2 & 27.8 & 29.3 & 27.4 \\
\hline & 9 & 3692 & 3700 & 3374 & 3589 & 29.2 & 33.1 & 30.3 & 30.9 \\
\hline & 18 & 4079 & 4102 & 4062 & 4081 & 36.8 & 34.8 & 37.1 & 36.2 \\
\hline & 27 & 5258 & 5210 & 4689 & 5052 & 38.8 & 37.2 & 35.8 & 37.3 \\
\hline & 36 & 7606 & 7640 & 8113 & 7786 & 30.0 & 34.8 & 35.3 & 33.4 \\
\hline \multirow[t]{6}{*}{1} & -3 & 4634 & 4633 & 4549 & 4605 & 20.2 & 12.7 & 21.3 & 18.0 \\
\hline & 3 & 2901 & 2876 & 2846 & 2874 & 26.3 & 24.5 & 25.2 & 25.3 \\
\hline & 9 & 2742 & 2886 & 2559 & 2729 & 34.0 & 35.3 & 35.1 & 34.8 \\
\hline & 18 & 3531 & 3739 & 3262 & 3511 & 37.5 & 39.1 & 37.6 & 38.1 \\
\hline & 27 & 4302 & 4401 & 4469 & 4391 & 37.6 & 35.0 & 38.5 & 37.0 \\
\hline & 36 & 6242 & 6115 & 5835 & 6064 & 40.4 & 37.3 & 38.1 & 38.6 \\
\hline \multirow[t]{6}{*}{0.5} & -3 & 3687 & 3682 & 3505 & 3625 & 22.3 & 18.4 & 17.3 & 19.3 \\
\hline & 3 & 2530 & 2514 & 2562 & 2535 & 30.0 & 26.7 & 27.3 & 28.0 \\
\hline & 9 & 2131 & 2246 & 2186 & 2188 & 32.2 & 31.9 & 35.3 & 33.1 \\
\hline & 18 & 2504 & 2503 & 2677 & 2561 & 37.7 & 35.7 & 37.5 & 37.0 \\
\hline & 27 & 3412 & 3509 & 3452 & 3458 & 36.9 & 38.5 & 35.5 & 37.0 \\
\hline & 36 & 4837 & 4791 & 4519 & 4716 & 38.5 & 37.7 & 38.5 & 38.2 \\
\hline \multirow[t]{6}{*}{0.3} & -3 & 3462 & 2880 & 3161 & 3168 & 18.0 & 16.0 & 19.6 & 17.9 \\
\hline & 3 & 2146 & 2235 & 2195 & 2192 & 33.2 & 31.6 & 36.2 & 33.7 \\
\hline & 9 & 1797 & 1918 & 2131 & 1949 & 35.7 & 33.4 & 35.4 & 34.8 \\
\hline & 18 & 2356 & 2346 & 2345 & 2349 & 46.9 & 46.3 & 45.6 & 46.3 \\
\hline & 27 & 3305 & 2776 & 3435 & 3172 & 45.0 & 46.8 & 45.6 & 45.8 \\
\hline & 36 & 4722 & 4700 & 4487 & 4636 & 44.0 & 45.0 & 45.0 & 44.7 \\
\hline
\end{tabular}

Table A.2. Tilted U-notched samples (reproduced from [2]).

\begin{tabular}{|c|c|c|c|c|c|c|c|c|c|}
\hline$\rho(\mathrm{mm})$ & $m(\mathrm{~mm})$ & $P_{1}(\mathrm{~N})$ & $P_{2}(\mathrm{~N})$ & $P_{3}(\mathrm{~N})$ & $\langle P\rangle(\mathrm{N})$ & $\varphi_{1}^{\circ}$ & $\varphi_{2}^{\circ}$ & $\varphi_{3}^{\circ}$ & $\langle\varphi\rangle^{\circ}$ \\
\hline \multirow[t]{3}{*}{4} & 15 & 3433 & 3329 & 3466 & 3409 & 64.1 & 64.8 & 63.9 & 64.3 \\
\hline & 9 & 4225 & 4209 & 4448 & 4294 & 67.2 & 66.6 & 68.7 & 67.5 \\
\hline & 3 & 6613 & 6337 & 6353 & 6434 & 77.8 & 78.5 & 76.6 & 77.6 \\
\hline \multirow[t]{3}{*}{2} & 15 & 2842 & 2809 & 3112 & 2921 & 66.4 & 64.9 & 66.5 & 65.9 \\
\hline & 9 & 3743 & 3854 & 3729 & 3775 & 67.5 & 68.5 & 76.7 & 70.9 \\
\hline & 3 & 6316 & 6086 & 5914 & 6105 & 84.5 & 85.9 & 83.6 & 84.7 \\
\hline \multirow[t]{3}{*}{1} & 15 & 2266 & 2310 & 2460 & 2345 & 63.7 & 63.1 & 61.8 & 62.9 \\
\hline & 9 & 3327 & 3164 & 3008 & 3166 & 69.3 & 65.9 & 69.2 & 68.1 \\
\hline & 3 & 5435 & 4851 & 5316 & 5201 & 77.2 & 84.3 & 80.3 & 80.6 \\
\hline \multirow[t]{3}{*}{0.5} & 15 & 1792 & 1783 & 1776 & 1784 & 64.5 & 64.1 & 63.7 & 64.1 \\
\hline & 9 & 2323 & 2494 & 2436 & 2418 & 68.4 & 70.3 & 67.9 & 68.9 \\
\hline & 3 & 4503 & 4732 & 4334 & 4523 & 83.3 & 84.8 & 87.5 & 85.2 \\
\hline \multirow[t]{3}{*}{0.3} & 15 & 1736 & 1866 & 1669 & 1757 & 60.4 & 62.0 & 60.6 & 61.0 \\
\hline & 9 & 2397 & 2420 & 2446 & 2421 & 72.6 & 68.1 & 74.2 & 71.6 \\
\hline & 3 & 4394 & 4354 & 4173 & 4307 & 86.2 & 87.0 & 85.5 & 86.2 \\
\hline
\end{tabular}



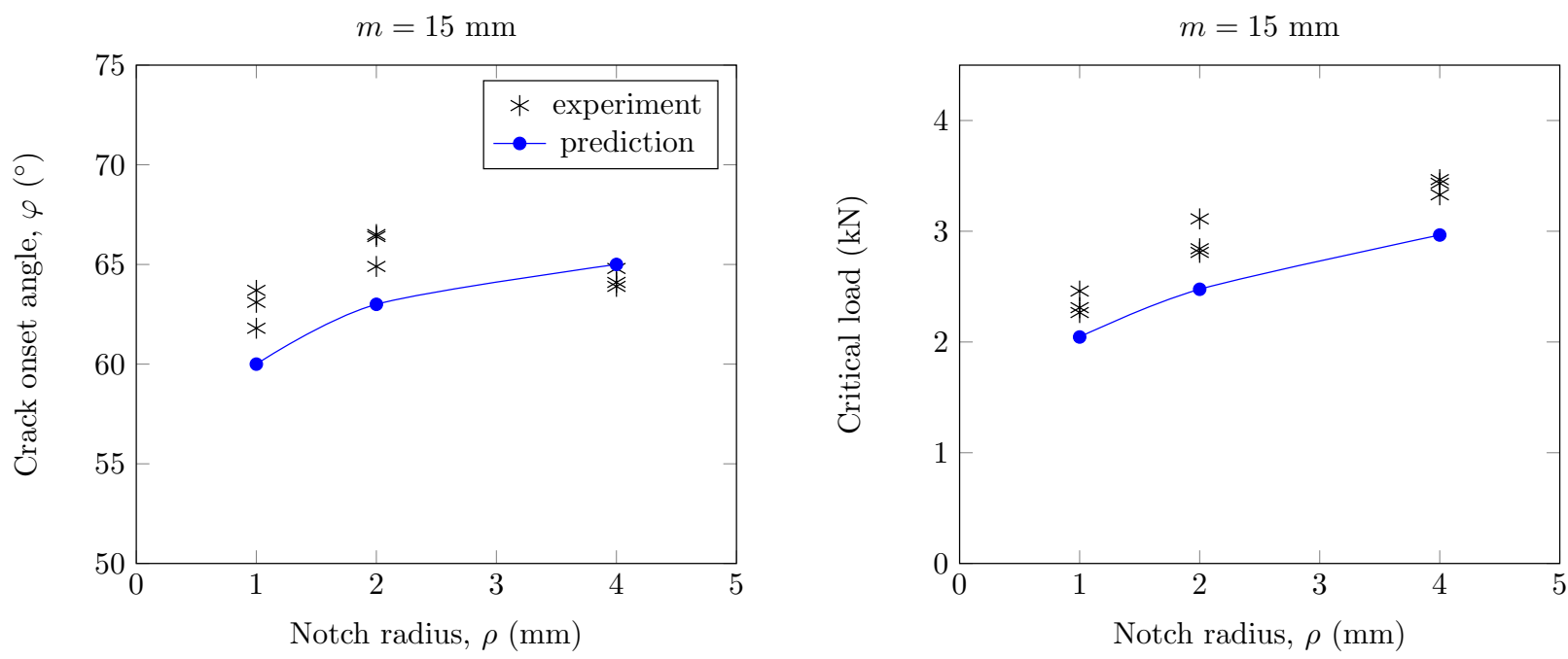

Figure 12. Experimental results and numerical predictions for $m=15 \mathrm{~mm}$.

\section{References}

[1] F.J. Gómez, M. Elices, F. Berto, and P. Lazzarin. Local strain energy to assess the static failure of U-notches in plates under mixed mode loading. International Journal of Fracture, 145(1):29-45, 2007.

[2] F.J. Gómez, M. Elices, F. Berto, and P. Lazzarin. Fracture of U-notched specimens under mixed mode: Experimental results and numerical predictions. Engineering Fracture Mechanics, 76(2):236-249, 2009.

[3] M. Elices, G.V. Guinea, J. Gómez, and J. Planas. The cohesive zone model: advantages, limitations and challenges. Engineering Fracture Mechanics, 69(2):137-163, 2002.

[4] F.J. Gómez, M. Elices, and J. Planas. The cohesive crack concept: application to PMMA at $-60^{\circ}$ C. Engineering Fracture Mechanics, 72(8):1268-1285, 2005.

[5] F. Berto, P. Lazzarin, F.J. Gómez, and M. Elices. Fracture assessment of U-notches under mixed mode loading: two procedures based on the 'equivalent local mode I' concept. International Journal of Fracture, 148(4):415-433, 2007.

[6] V.V. Novozhilov. On a necessary and sufficient criterion for brittle strength: PMM Vol. 33, $\mathrm{N}^{\circ} 2,1969$, pp. $212-222$. Journal of Applied Mathematics and Mechanics, 33(2):201-210, 1969.

[7] Nicola M. Pugno and Rodney S. Ruoff. Quantized fracture mechanics. Philosophical Magazine, 84(27):2829-2845, 2004.

[8] Andrzej Seweryn and Zenon Mróz. A non-local stress failure condition for structural elements under multiaxial loading. Engineering Fracture Mechanics, 51(6):955-973, 1995.

[9] A. Seweryn, S. Poskrobko, and Z. Mróz. Brittle fracture in plane elements with sharp notches under mixed-mode loading. Journal of Engineering Mechanics, 123(6):535-543, 1997.

[10] A. Seweryn and A. Łukaszewicz. Verification of brittle fracture criteria for elements with V-shaped notches. Engineering Fracture Mechanics, 69(13):1487-1510, 2002.

[11] C. Kodsi. Crack initiation: A non-local energy approach. Engineering Fracture Mechanics, 165:153-182, 2016.

[12] C. Kodsi. Computational Framework for Fracture of Graphite Bricks in an AGR Core. PhD thesis, University of Glasgow, 2017.

[13] Alan A. Griffith. The phenomena of rupture and flow in solids. Philosophical Transactions of the Royal Society of London. Series A, 221(582-593):163-198, 1921.

[14] J. Li and X.B. Zhang. A criterion study for non-singular stress concentrations in brittle or quasi-brittle materials. Engineering Fracture Mechanics, 73(4):505-523, 2006.

[15] De Soza T. Code_Aster: guide de lecture de la documentation de référence. http://www.code-aster.org, 2013.

[16] F. Berto, P. Lazzarin, M. Elices, and F.J. Gómez. Brittle failure and crack initiation angle in plates weakened by V- and U-notches under mixed mode (I+II) loading. In CP2009.

[17] AR Torabi, M Fakoor, and E Pirhadi. Fracture analysis of U-notched disc-type graphite specimens under mixed mode loading. International Journal of Solids and Structures, 51(6):1287-1298, 2014. 